$\begin{array}{cc}\text { ORIENTAL JOURNAL OF } & \text { ISSN: 0974-6471 } \\ \text { December 2016, } \\ \text { Vol. 9, No. (3): } \\ \text { Pgs. 204-211 }\end{array}$

\title{
Improving WLAN Quality of Services (Qos) Using Opnet
}

\author{
ISHWAR BAIDARI ${ }^{1}$, S.P. SAJJAN ${ }^{2}$ and AJEET KUMAR SINGH ${ }^{2}$ \\ ${ }^{1}$ Karnatak University of Dharwad-03, India. \\ ${ }^{2}$ Central University of Karnataka-67, India. \\ http://dx.doi.org/10.13005/ojcst/09.03.06
}

(Received: March 16, 2016; Accepted: May 20, 2016)

\begin{abstract}
Wireless local area networks (WLANs) are in a period of great expansion and there is a strong need for them to support multimedia applications. With the increasing demand and penetration of wireless services, users of wireless networks now expect Quality of Service (QoS) and performance comparable to what is available from fixed networks. Providing QoS requirements like good throughput and minimum access delay are challenging tasks with regard to 802.11 WLAN protocols and Medium Access Control (MAC) functions. This research is done to study, the presently implemented schemes (the Point Coordination Function (PCF) of IEEE 802.11, the Enhanced Distributed Coordination Function (EDCF) of the proposed IEEE 802.11e extension to IEEE 802.11), solves these issues and what can be done to improve them further. The metrics used were Throughput, Data Drop, Retransmission and Medium Access Delay, to analyze the performance of various MAC protocols in providing QoS to users of WLAN. Two scenarios, with same Physical and MAC parameters, one implementing the DCF and other EDCF, were created in the network simulation tool (OPNET MODELER) to obtain the results. The results showed that the performance of EDCF was better in providing QoS for real-time interactive services (like video conferencing) as compared to DCF, because of its ability to differentiate and prioritize various services. Index Terms - Wireless local area networks (WLANs),
\end{abstract}

Keywords: Expect Quality of Service (QoS), Point Co-ordination Function (PCF), Enhanced Distributed Coordination Function (EDCF), OPNET.

\section{INTRODUCTION}

As the technology is improving so are the demands of end users and their applications increasing. A wide variety of new applications are being invented daily. High bandwidth internet connectivity has become a basic requirement to the success of almost all of these areas. Wireless Local Area Networks (WLANs) has become one of the most promising and successful technology in recent years. WLANs provide free wireless connectivity to end users, offering an easy and viable access to the network and its services. Wireless networks are superior to wired networks with regard to aspects such as ease of installation and flexibility. They do, however, suffer from lower bandwidth, higher delays, higher bit-error rates, and higher costs than wired networks. 
With the advent of Wireless Local Area Networks (WLANs), bandwidth has increased and prices havedecreased on Wireless networking solutions. These factors have made WLANs a very popular Wireless networking solution.

\section{Working of Wlan}

Wireless networks perform functions similar to their wired Ethernet. Networks perform the following functions to enable the transfer of information from source to destination':

1. The medium provides a bit pipe (a path for data to flow) for the transmission of data.

2. Medium access techniques facilitate the sharing of a common medium.

3. Synchronization and error control mechanisms ensure that each link transfer the data intact.

4. Routing mechanisms move the data from the originating source to the intended destination.

5. Connectivity software interfaces an appliance, such as a pen-based computer or bar code scanner, to application software hosted on a server.

\section{Advantage of Wireless Lan Flexibility}

Within radio coverage, nodes can communicate without further restriction. Radio waves can penetrate walls. Planning: wireless ad hoc networks allow for communication without planning. Wired networks need wiring plans. Robustness: wireless networks can survive disasters; if the wireless devices survive people can still communicate.

\section{Disadvantages of Wlan}

Connectivity: There are no wires to connect to the Wi-Fi network but then the area of the hotspot is very limited and if the node gets out of the area it will be disconnected. Bandwidth Coverage: This is perhaps the greatest disadvantages you have to be within 100-150 ft of the base station (indoors) and about 100-300 meters (outdoors) to get connected. QoS(Quality of Service): WLANs offer typically lower QoS. Lower bandwidth due to limitations in radio transmission and higher error rates due to interference.

\section{Preliminaries of Wlan}

Logical Architecture Of Wlan:Wlan works in the lower two layers of OSI model. First one is the physical layer which takes care of transmission of bits through a communication channel by defining electrical, mechanical, and procedural specifications. Second one is the data link layer which is sub-divided into two layers: logical link layer (LLC) and Medium Access Control layer (MAC) [1] Only MAC layer is considered as the part of wireless LAN Functions.

Medium Access Control (Mac) Sub Layer: The primary function of a MAC protocol is to define a set of rules and give the stations a fair access to the channel for successful communication. Many MAC protocols provide the standardized medium access and physical layer protocols for WLANs and it is the most widely employed standard in wireless networks.

Quality Of Service:Quality of Service (QoS) is the ability to provide a level of assurance for data delivery over the network. For example, traffic of different classes or traffic with different requirements receives different levels of QoS assurance.

Some of factors that influence QoS of Wireless Network include:-

\section{Throughput of Network}

Represents the total number of bits (in bits/ sec) forwarded from wireless LAN layers to higher layers in all WLAN nodes of the network.

\section{Retransmission Attempts}

Total number of retransmission attempts by all WLAN MACs in the network until either packet is successfully transmitted or it is discarded as a result of reaching short or long retry limit.

\section{Data Dropped}

Data dropped due to unavailability of access to medium.

\section{Medium Access Delay}

It includes total of queuing and contention delays of the data. 


\section{Problem Definition}

High raw data rates (up to $54 \mathrm{Mbps}$ as per standards and twice that in proprietary ways) at physical layer have become possible in wireless communication. A MAC protocol should provide an efficient use of the available bandwidth while satisfying the Quality of Service (QoS) requirements of both data and real-time applications. Real-time services such as streaming voice and video require a certain quality of service such as low packet loss and low delay to perform well. To provide QoS for such kind of application, service differentiation is must. Service differentiation means that different types of traffic have different requirements on the network. Various mechanisms have been developed to support quality of service but no effort has been made to implement the latest techniques in OPNET Modeler and compare them in order to know

Table 1

\begin{tabular}{|c|c|}
\hline Number & Topic \\
\hline 802.1 & $\begin{array}{c}\text { Overview and architecture } \\
\text { of LANs }\end{array}$ \\
\hline $802.2 \downarrow$ & Logical link control \\
\hline 802.3 & Ethernet \\
\hline $802.4 \downarrow$ & $\begin{array}{c}\text { Token bus(was briefly used in } \\
\text { manufacture plants }\end{array}$ \\
\hline 802.5 & $\begin{array}{l}\text { Token ring(IBM's entry into the } \\
\text { LAN world) }\end{array}$ \\
\hline $802.6 \downarrow$ & $\begin{array}{l}\text { Dual queue dual bus(early } \\
\text { metropolitan area network) }\end{array}$ \\
\hline $802.7 \downarrow$ & $\begin{array}{l}\text { Technical advisory group on } \\
\text { broadband technologies }\end{array}$ \\
\hline $802.8 \uparrow$ & $\begin{array}{l}\text { Technical advisory group on } \\
\text { fiber optic technologies }\end{array}$ \\
\hline $802.9 \downarrow$ & $\begin{array}{l}\text { Isochronous LANs(for real-time } \\
\text { application) }\end{array}$ \\
\hline $802.10 \downarrow$ & Virtual LANs and security \\
\hline 802.11 & Wireless LANs \\
\hline $802.12 \downarrow$ & $\begin{array}{l}\text { Demand priority (Hewlett - } \\
\text { Packard's Any LAN) }\end{array}$ \\
\hline 802.13 & $\begin{array}{l}\text { Unlucky number. Nobody } \\
\text { wanted it }\end{array}$ \\
\hline $802.14 \downarrow$ & $\begin{array}{c}\text { Cable modems (defunct: an industry } \\
\text { consortium got there first) }\end{array}$ \\
\hline 802.15 . & Personal area networks(Bluetooth) \\
\hline 802.16 . & Broadband wireless \\
\hline 802.17 & Resilient packed ring \\
\hline
\end{tabular}

which one is the best under which type of traffic condition.

\section{Objective}

1. The study of basic concepts and issues of Wireless/Cellular network that can improve the QoS of a cellular WLAN. Mainly focusing on Medium Access Control layer of Open Systems Interconnection (OSI) model.

2. Study various existing Medium Access Control protocols of cellular WLAN.

3. Implementation and comparison of efficient mechanisms that can improve the QoS of WLAN by using OPNET Modeler's wireless module.

4. Study the results obtained, and recommend the best possible protocol that can

5. Provide high QoS under respective network traffic conditions.

\section{IEEE 802 Standards Working Groups IEEE 802.11(Wireless LANs)}

The following IEEE 802.11 standards exist or are in development to support the creation of technologies for wireless local area networking:

1. $802.11 \mathrm{a}-54 \mathrm{Mbps}$ standard, $5 \mathrm{GHz}$ signaling (ratified 1999)

2. $802.11 \mathrm{~b}$ - $11 \mathrm{Mbps}$ standard, $2.4 \mathrm{GHz}$ signaling (1999)

3. $802.11 \mathrm{c}$ - operation of bridge connections (moved to 802.1D)

4. 802.11d - worldwide compliance with regulations for use of wireless signal spectrum (2001)

5. 802.11e - Quality of Service (QoS) support (not yet ratified)

6. $\quad 802.11 \mathrm{f}$ - Inter-Access Point Protocol recommendation for communication between access points to support roaming clients (2003)

7. $\quad 802.11 \mathrm{~g}-54 \mathrm{Mbps}$ standard, $2.4 \mathrm{GHz}$ signaling (2003)

8. $802.11 \mathrm{~h}$ - enhanced version of $802.11 \mathrm{a}$ to support European regulatory requirements (2003)

9. $802.11 \mathrm{i}$ - security improvements for the 802.11 family (2004)

10. $802.11 \mathrm{j}$ - enhancements to $5 \mathrm{GHz}$ signaling to support Japan regulatory requirements 
(2004)

11. 802.11k - WLAN system management (in progress)

12. $802.11 \mathrm{~m}$ - maintenance of 802.11 family documentation

13. $802.11 n-100+$ Mbps standard improvements over $802.11 \mathrm{~g}$ (in progress)

14. 802.11p-Wireless Access for the Vehicular Environment

15. 802.11r - fast roaming support via Basic Service Set transitions

16. 802.11s - ESS mesh networking for access points

17. 802.11t - Wireless Performance Prediction - recommendation for testing standards and metrics

18. 802.11u - internetworking with 3G / cellular and other forms of external networks

19. 802.11v - wireless network management / device configuration

20. 802.11w - Protected Management Frames security enhancement

21. 802.11x-skipped (generic name for the 802.11 family)

22. 802.11y - Contention Based Protocol for interference avoidance

\subsection{MAC Sub layer Protocol}

- In 802.11 wireless LANs, "seizing the channel" does not exist as in 802.3 wired Ethernet.

- Two additional problems:

- Hidden Terminal Problem

- $\quad$ Exposed Station Problem

- $\quad$ To deal with these two problems 802.11 supports two modes of operation:

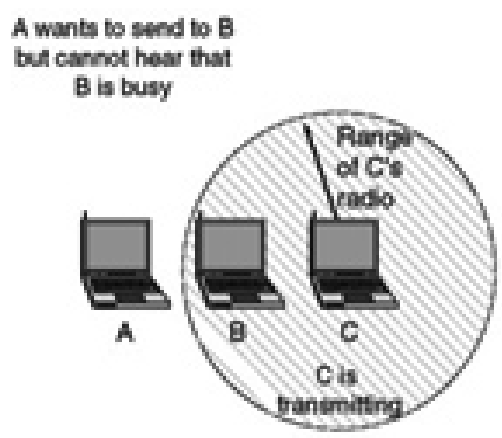

(a)
- $\quad$ DCF (Distributed Coordination Function)

- PCF (Point Coordination Function).

All implementations must support DCF, but PCF is optional.

\section{The Hidden Terminal Problem}

1. Wireless stations have transmission ranges and not all stations are within radio range of each other.

2. Simple CSMA will not work!

3. $C$ transmits to $B$.

4. If A "senses"the channel, it will not hear C's transmission and falsely conclude that A can begin a transmission to $B$.

\section{The Exposed Station Problem}

1. This is the inverse problem.

2. B wants to send to $C$ and listens to the channel.

3. When B hears A's transmission, B falsely assumes that it cannot send to $\mathrm{C}$.

\section{Point Coordinated Function (PCF)}

1. PCF uses a base station to poll other stations to see if they have frames to send.

2. No collisions occur.

3. Base station sends beacon frame periodically.

4. Base station can tell another station to sleep to save on batteries and base stations holds frames for sleeping station.

5. DCF and PCF Co-Existence

6. Distributed and centralized control can coexist using InterFrame Spacing.

7. SIFS (Short IFS) :: is the time waited between packets in an ongoing dialog (RTS,CTS,data, ACK, next frame)

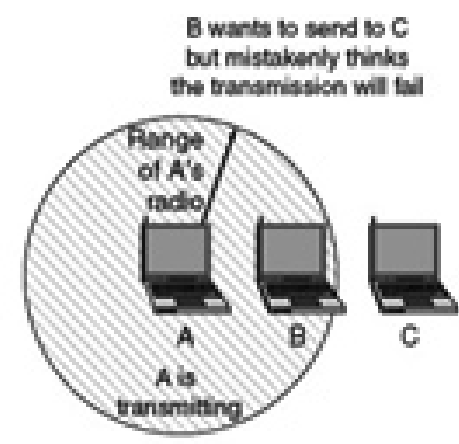

(b) 
Interframe Spacing in $\mathbf{8 0 2 . 1 1}$

- $\quad$ PIFS (PCF IFS) :: when no SIFS response, base station can issue beacon or poll.

- $\quad$ DIFS (DCF IFS) :: when no PIFS, any station can attempt to acquire the channel.

- $\quad$ EIFS (Extended IFS) :: lowest priority interval used to report bad or unknown frame.

\section{Introduction To Opnet}

OPNET MODELER is used to design and study communication networks, devices, protocols and applications. It provides a graphical editor interface to build models for various network entities from physical layer modulator to application processes.

\section{OPNET supports model specification with a} number of tools, called editors.

1. Project Editor

2. Node Editor

3. Process Editor

4. External System Editor

5. Link Model Editor

6. Packet Format Editor

7. Link Editor

\section{Run Simulation}

1. Discrete Event Simulation

2. Flow Analysis

3. Failure Impact Analysis

4. Net Doctor Validation

\section{Discrete Event Simulation}

Discrete event simulation provides the most detailed results but has the longest running times. This is because it does a more thorough analysis than the others, handling explicit traffic, conversation pair traffic, and link loads. The other types answer specific types of questions and generate results much faster than a discrete event simulation. A flow analysis, for example, handles only conversation pair traffic (flows) and a Net Doctor validation does not consider traffic at all. Licenses for generating Discrete Event Simulations (DES) are available for this studies and analysis.

\section{Distributed Co-Ordination Function (DCF)}

Distributed Coordination Function is the basic access mechanism used in IEEE 802.11.It uses a Carrier Sense Multiple Access with Collision Avoidance (CSMA/CA) algorithm to mediate the access to the shared medium. Before discussing DCF, there is terminology used in technicalities that one needs to know; that are as follows:

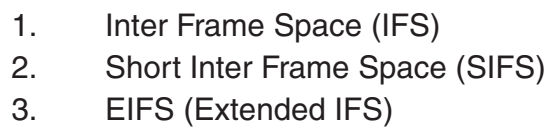

$C W[i]=2(k+i)-1$

Where $\mathrm{i}$ is the number of attempts (including the current one) to transmit the frame that has been done, and $\mathrm{k}$ is a constant defining the minimum contention window, $\mathrm{CWmin}=2 \mathrm{k}$ "1. A new backoff time is then chosen and the backoff procedure starts over. The backoff mechanism is also used after a successful transmission before sending the next frame. After a successful transmission, the contention window is reset to CWmin.

\section{Enhanced Distributed Coordination Function (EDCF)}

EDCF is designed to provide prioritized QoS by enhancing the contention-based DCF. It provides differentiated, distributed access to the

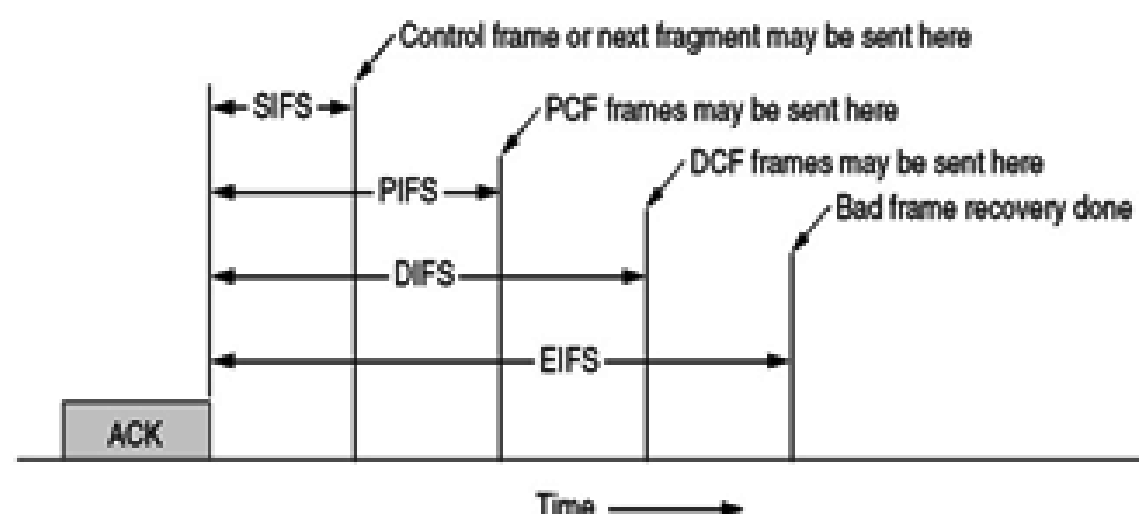


wireless medium for QoS stations (QSTAs) using 8 different user priorities (UPs). Before entering the MAC layer, each data packet received from the higher layer is assigned a specific user priority value.
The EDCF mechanism defines four different first-in first-out (FIFO) queues, called access categories (ACs) that provide support for the delivery of traffic with UPs at the QSTAs. Each data packet from the

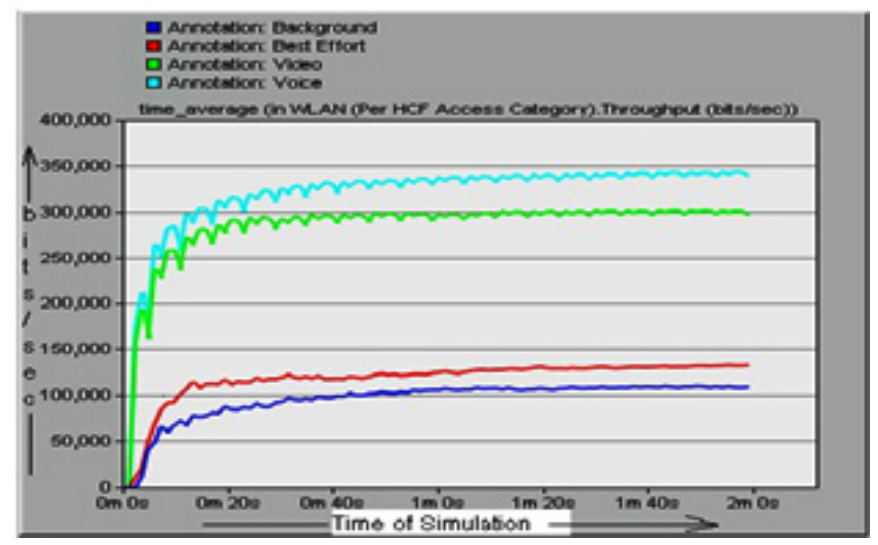

Fig. 1: Throughput of Different Access Categories

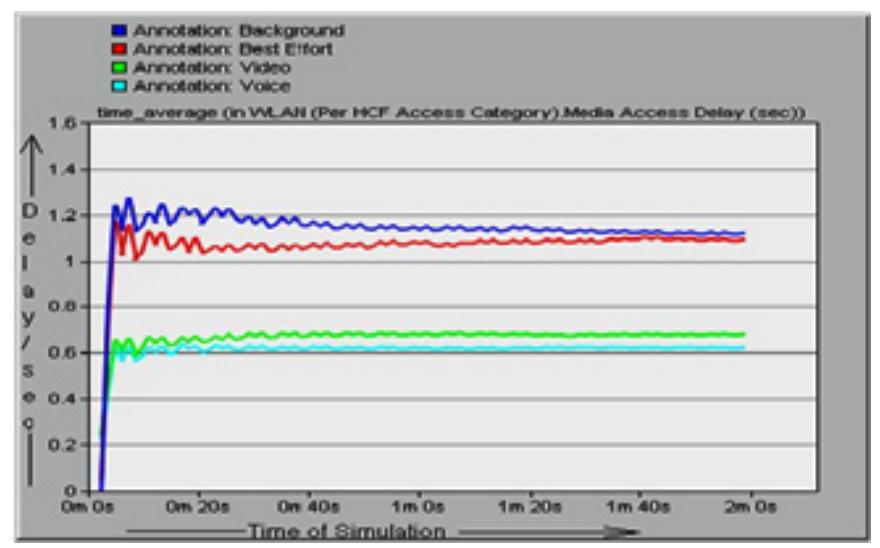

Fig. 2: WLAN Media Access Delay

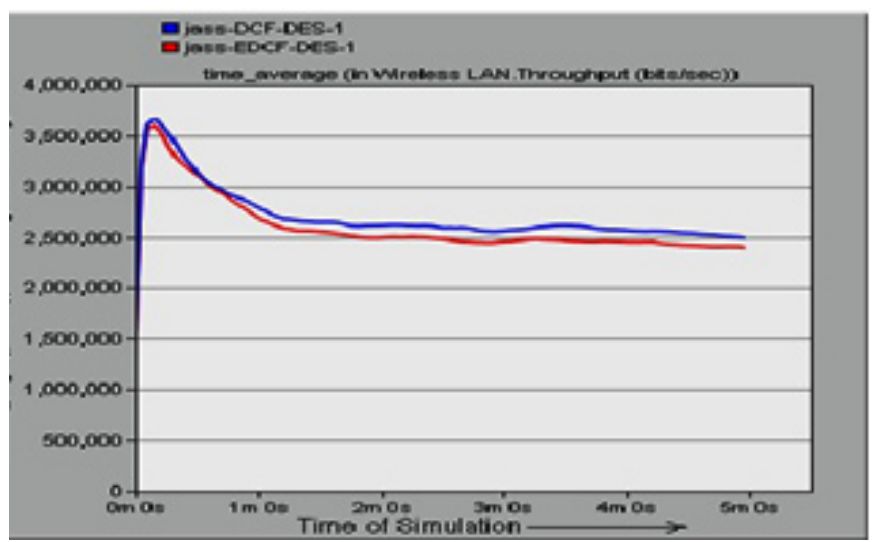

Fig. 3: Throughput of DCF versus EDCF 


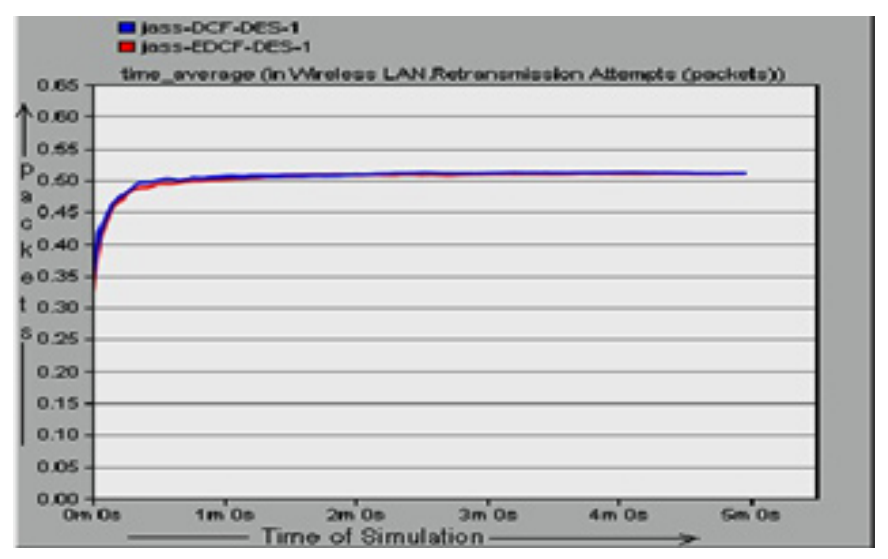

Fig. 4: Retransmission Attempts DCF vs EDCF

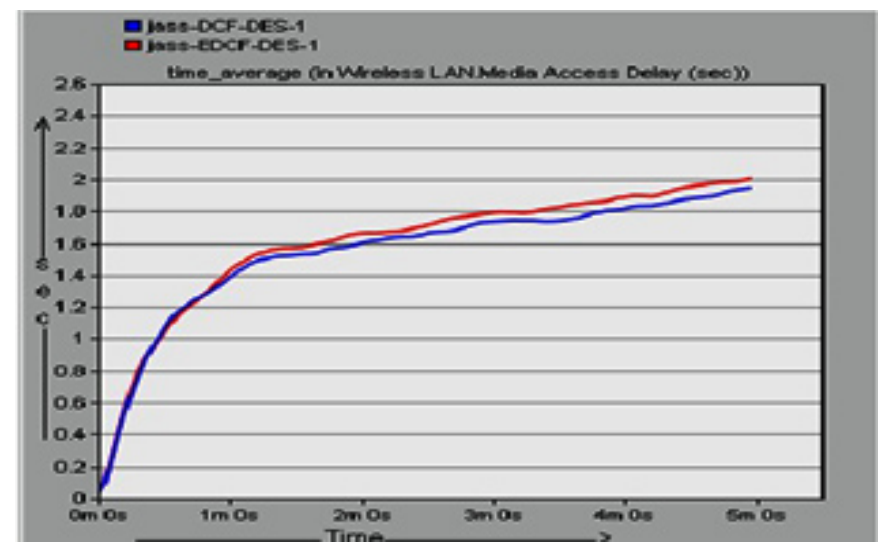

Fig. 5: Media Access Delay of DCF versus EDCF

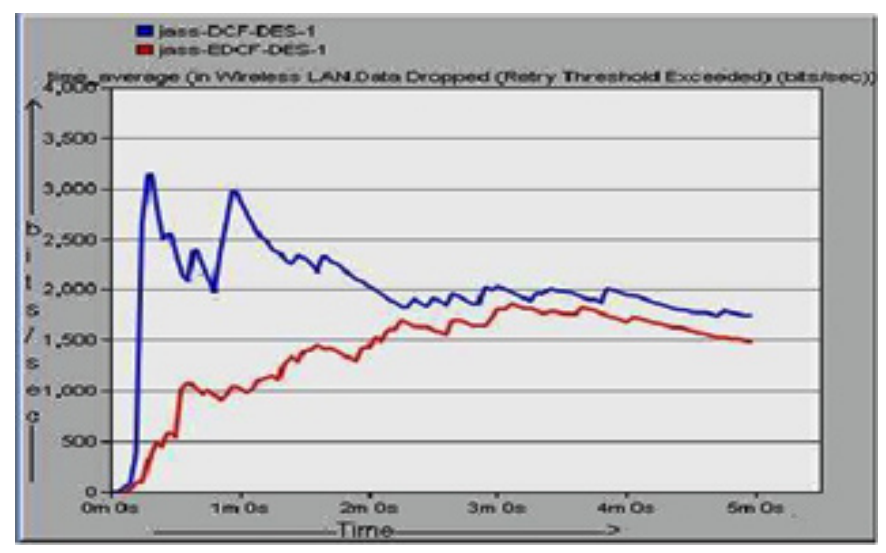

Fig. 6: Amount of Data Dropped DCF versus EDCF 
higher layer along with a specific user priority value should be mapped into a corresponding.

$$
\text { AIFS }[A C]=\text { SIFS }+ \text { AIFSN }[A C] \times \text { Slot Time }
$$

Where

AIFS Arbitration Inter-frame Space

\section{SIFSShort Inter Frame Space}

AIFSN $[A C]$ is called the arbitration IFS number determined by the AC physical settings and the duration of a time slot. Throughput Of Different Access Categories

\section{CONCLUSION}

The results obtained from simulation shows that Enhanced Distribution Coordination Function provides efficient mechanism for service differentiation and hence provides quality of service to the Wireless LAN. However, this improvement comes at a cost of a decrease in quality of the lower priority traffic up to the point of starvation. The acquisition of the radio channel by the higher priority traffic is much more aggressive than for the lower priority. Higher priority traffic benefited, while lower priority traffic suffered. In terms of overall performance (under the used simulation conditions in this particular study of QoS of Wireless LAN), DCF performs marginally well than EDCF. This happens due to reason that in EDCF mechanism, each $A C$ function acts like a virtual station for medium access, so more collision will be expected for EDCF scenario. But in terms of Quality of Service for real-time applications (like Video conferencing) EDCF outperforms DCF. EDCF has been purposed as the medium access control protocol for IEEE's upcoming standard IEEE 802.11e. The wireless devices using EDCF as MAC protocol would be available in market in the next coming two years. Presently, all of the wireless devices use DCF as the default MAC protocol and PCF as the optional functionality.

\section{REFERENCES}

1. Choi Sunghyun, Del, Sai, Mangold S. "IEEE 802.11 e contention-based channel access (EDCF) performance evaluation", IEEE International Conference on In Communications, 2, pp. 1151-1156, (2003).

2. Choi S. and Shin K. G., "A cellular local area network with QoS guarantees for heterogeneous traffic", Proceedings IEEE Infocom'97, pp. 1032-1039, Kobe, Japan, (1997).

3. Crow Brian P., Widjajalndra, Kim JeongGeun, Prescott T. Sakai,"IEEE 802.11 Wireless Local Area Networks", IEEE Communications Magazine, (1997).

4. Draft Standard IEEE 802.11, "Wireless LAN Medium Access Control (MAC) and Physical Layer (PHY) Specifications", (1999).

5. Grilo A. and Nunes M., "Performance Evaluation of IEEE 802.11e", In Proc. IEEE Personal, Indoor and Mobile Radio
Communications (PIMRC), vol. 1, pp. 511 -517, (2002).

6. Gu D. and Zhang J., "QoS enhancement in IEEE 802.11 wireless local area networks", IEEE Communications Magazine, vol. 41, no. 6, pp. 120 -124, (2003).

7. Hannikainen Marko, LavikkoTommi, Kukkala Petri And HamalainenTimo D., QoS Supporting Wireless Network", published in Kluwer Academic Publishers., (2003).

8. Kim Andrew, "OPNET Tutorial", TECOMM LAB, (2003).

9. Lindgren Anders, Almquist Andreas, and SchelenOlov,"Quality of Service Schemes for IEEE 802.11" (2001).

10. Liu X., Chong E. K. P., and Shroff N. B., "Opportunistic Scheduling with QoS Requirements in Time-varying Wireless Channels", Computer Networks, 4(41): pp. 451-474 (2003). 